\title{
Cascaded Dilated Deep Residual Network for Volumetric Liver Segmentation From CT Image
}

\author{
Gajendra Kumar Mourya, North-Eastern Hill University, Shillong, India \\ iD https://orcid.org/0000-0003-0585-4964 \\ Manashjit Gogoi, North-Eastern Hill University, Shillong, India \\ iD https://orcid.org/0000-0002-1753-192X
}

S. N. Talbar, Shri Guru Gobind Singhji Institute of Engineering and Technology, Nanded, India

Prasad Vilas Dutande, Shri Guru Gobind Singhji Institute of Engineering and Technology, Nanded, India

Ujjwal Baid, Shri Guru Gobind Singhji Institute of Engineering and Technology, Nanded, India

\begin{abstract}
Volumetric liver segmentation is a prerequisite for liver transplantation and radiation therapy planning. In this paper, dilated deep residual network (DDRN) has been proposed for automatic segmentation of liver from CT images. The combination of three parallel DDRN is cascaded with fourth DDRN in order to get final result. The volumetric CT data of 40 subjects belongs to "Combined Healthy Abdominal Organ Segmentation" (CHAOS) challenge 2019 is utilized to evaluate the proposed method. Input image converted into three images using windowing ranges and fed to three DDRN. The output of three DDRN along with original image fed to the fourth DDRN as an input. The output of cascaded network is compared with the three parallel DDRN individually. Obtained results were quantitatively evaluated with various evaluation parameters. The results were submitted to online evaluation system, and achieved average dice coefficient is $0.93 \pm 0.02$; average symmetric surface distance (ASSD) is $4.89 \pm 0.91$. In conclusion, obtained results are prominent and consistent.
\end{abstract}

\section{KEYWORDS}

Computed Tomography, Convolutional Neural Network, Dilated Deep Residual Network, Dilation, Dilation Convolution Filter, Semantic Segmentation, Windowing

\section{INTRODUCTION}

The liver is the largest internal organ in human body, which is situated right side of the abdominal region. This organ performs the second-largest number of functions, such as detoxification of chemicals, drug metabolization and bile secretion (Glenisson et al., 2014; Thapa \& Walia, 2007). According to WHO, about $46 \%$ of global diseases, $59 \%$ of the mortality is because of chronic liver diseases(WHO, 2012). Liver diseases are major medical problems and mortality reached 216,865 or $2.44 \%$ of the total deaths in India (Asrani, Devarbhavi, Eaton, \& Kamath, 2019).

The most commonly Computed Tomography (CT), Ultrasonography (US), Magnetic resonance imaging (MRI) are used for early prognosis and analysis of anatomical abnormality. CT has often 
favored modality for the identification of different cancers because it provides high contrast, highresolution and image acquisition is faster compared to other medical imaging modalities (Beutel, Kundel, \& Van Metter, 2000). Liver segmentation helps the oncologist to measure the volume of the liver from 3D medical images in radiation dose planning. Liver volume estimation is the most difficult and challenging task among researchers. Since liver and its neighboring organs have approximately similar gray level intensity so it is difficult to delineate the liver boundary precisely (Figure 1 (a)) and liver divided into two parts (Figure 1 (b)).

Figure 1. Example of liver segmentation challenges (Axial View of 3D CT Scan) (a) Liver and heart intensity homogeneity highlighted by star (b) Liver divided into two parts highlighted by star

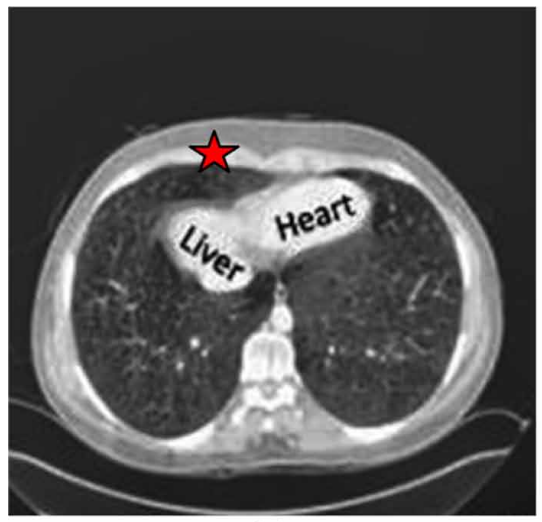

(a)

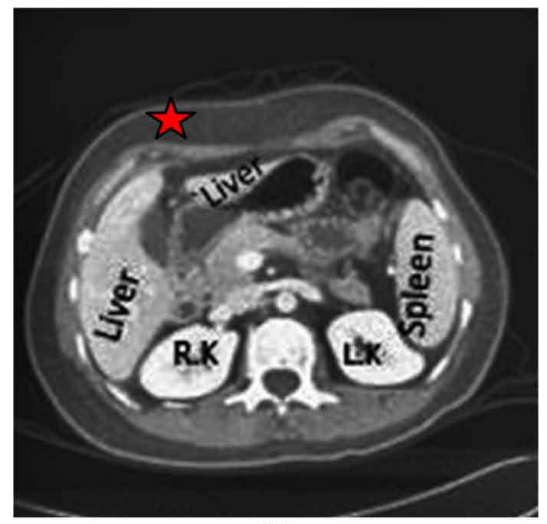

(b)

Windowing is one of the contrast enhancement methods which perform gray level slicing to each pixel in an image. A medical expert has to set the window settings to observe pathology within an image. Each window shows a different appearance in terms of varying contrast. Windowing is a subjective matter which changes from person to person. Therefore same idea is used while training the network architecture for different windows and its aggregation. Literature shows a single-window used for training the deep learning model, which has limited learning capability. Different windowing represents an image at various gray-level quantization and dilation convolution enhance learning capability at various spatial resolution of an input image. The proposed method is an amalgam of dilation convolution and windowing method along with a unique network connection.

Convolutional neural network $(\mathrm{CNN})$ is a combination of various building blocks such as convolutional layer, pooling layer and fully connected layer ( $\mathrm{Li}, \mathrm{Jia}, \& \mathrm{Hu}, 2015)$. The CNN becomes dominant in semantic segmentation for medical image analysis. Residual network (Res-Net) is one of variants of CNN. It helps to increase the depth of network along with learnable parameters without increasing the computational complexity. Dilation convolution extract features correspond to adjacent neighbor pixels and interval neighbor pixels as per the dilation rate.

In this paper, we are proposing a Cascaded DDRN for the automatic segmentation of liver. Three similar DDRN are connected in parallel and trained separately for different windows. The outputs of three DDRN combined with original image and feeds through the fourth cascaded DDRN. All DDRN have same architecture and learnable parameters. The proposed method achieved better performance while comparing it with evaluation parameters. This novel cascading technique is able to remove false-positive results from predicted maps and achieved substantial Average Symmetric Surface Distance (ASSD), Hausdorff Distance 95 (HD 95) evaluation values. 


\section{BACKGROUND}

Many researchers followed Deep Convolutional Neural Network (DCNN) trend and utilized CNN based models for medical image applications such as the liver and lesion segmentation. (Ben-Cohen, Diamant, Klang, Amitai, \& Greenspan, 2016) proposed a Fully Convolutional Network (FCN) based algorithm for semantic segmentation of liver and prognosis in CT images. (Shelhamer, Long, \& Darrell, 2017) developed FCN trained as end-to-end, pixel-to-pixel classification. It can accept random size images as input and produce fixed-size image as output. The VGG-16 net was very appealing because of its uniformity of layers throughout the network architecture (Simonyan \& Zisserman, 2014). The Res-net was introduced by (He, Zhang, Ren, \& Sun, 2016) reformulating the VGG net in order to decrease complexity. These are the good classification network produced efficient results on non-medical image datasets.

(Lateef \& Ruichek, 2019) provided a comprehensive survey on semantic segmentation methods using deep learning techniques and categorized as ten classes based on the architecture. Res-Net is placed in the class of "increase resolution features" along with various types of convolution. (Yu, Koltun, \& Funkhouser, 2017) presented module based on dilated convolutions to aggregate multiscale contextual information. They proved that dilated convolution help in the exponential expansion of the receptive field without loss of resolution or coverage. (Xue, Antani, Long, Demner-Fushman, $\&$ Thoma, 2012) describe the difficulty of the window selection in head CT to observe intra-cranial pathology. The proposed algorithm uses two classes of window settings and discusses how window setting can influence the classification results. U-net is one of the end-to-end pipeline and most captivated network for medical image segmentation (Ronneberger, Fischer, \& Brox, 2015). The conventional technique depends on edge enhancement techniques and post-processing operations (Mourya, Gogoi, \& Handique, 2020). Deep learning technique brings a breakthrough in the field of semantic segmentation, which, not required feature extraction and followed by classification. The U-Net modification took place for the automatic segmentation of liver from CT image (G K Mourya, S Paul, A Handique, U Baid, P V Dutande, n.d.). These modified networks tested on single windows and performance compared with the current state of the art. Volumetric liver segmentation remains challenging among scientists and still requires further modification in existing techniques(Conze et al., 2020).

\section{METHODOLOGY}

After a thorough literature review, we developed a novel procedure to get highly optimal results. The proposed workflow is depicted in Figure 2. The methodology contains three major steps. The first step deals with data preprocessing and preparation for the neural network segmentation. In a second step, cascaded Deep Dilated Residual Network for liver segmentation is discussed. In the final step, evaluation parameters are calculated to check the performance of proposed methodology. The proposed method implemented on CHAOS challenge dataset organized at ISBI-2019 (Bilic et al., 2019; "CHAOS - Combined (CT-MR) Healthy Abdominal Organ Segmentation," 2019). The dataset contains 40 subjects CT DICOM (Digital Imaging and Communications in Medicine) with physical parameters such as Image dimension 512x512, pixel spacing $0.65 \mathrm{~mm}$ to $0.79 \mathrm{~mm}$, slice thickness 1.6 to $3.2 \mathrm{~mm}$.

\section{Data Preprocessing}

The data preprocessing is carried out in a slice-wise fashion. Windowing is applicable for image pixel intensity, which represents 'CT Number.' Unsigned pixel intensity needs to convert into 'CT Number' using their slope and intercept value (equation -1), which is stored in DICOM header information. Windowing clips the ranges of CT numbers. In this paper CT Number windowed in the range [-200, $400],[-150,250]$ and $[-50,175]$ for each slice. Normalized slice feeds through DDRN. 
Figure 2. Schematic representations of the proposed methodology

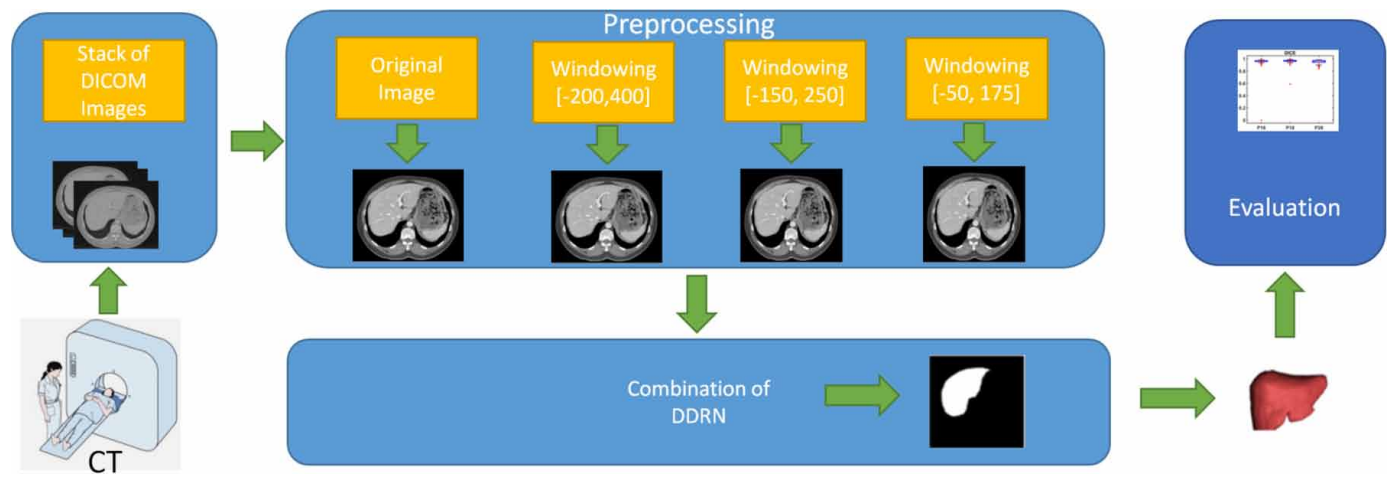

Figure 3. Three Types of residual connections used in proposed model (a) Direct Connection, (b) Projection Shortcut Connection, (c) Localization shortcut connection

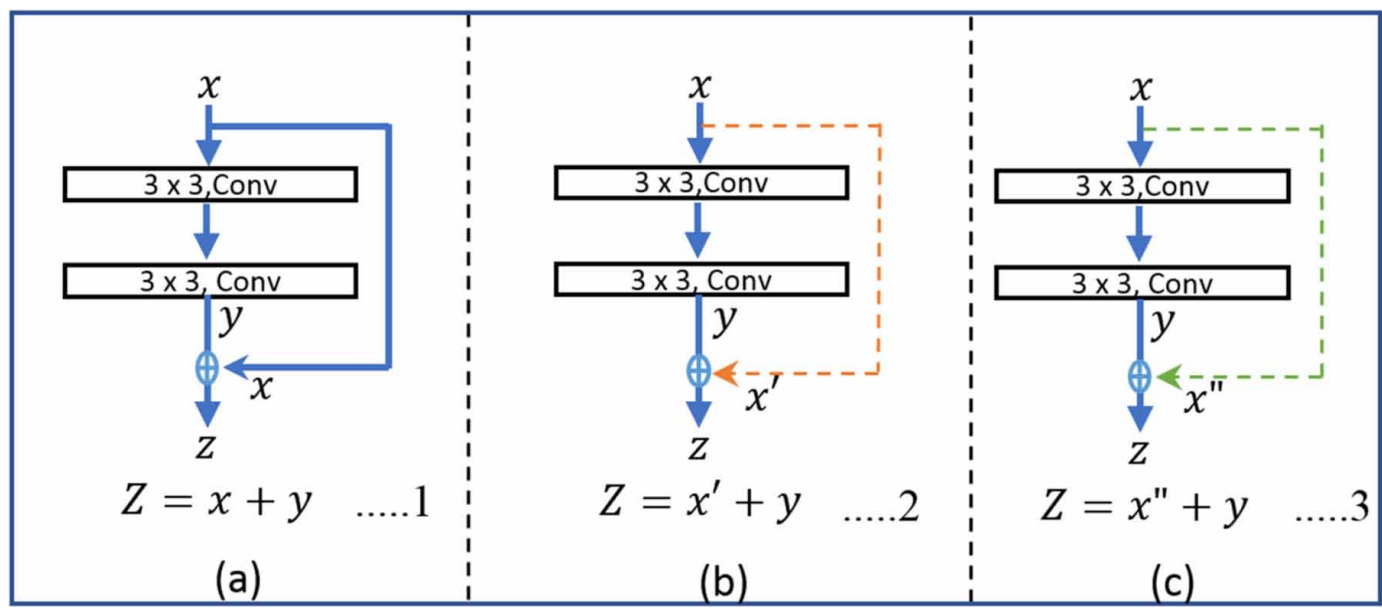

$Y_{C T}=U_{n} * S_{l}+I_{\text {intercept }}$

Where $\mathrm{Y}_{\mathrm{CT}}=$ CT Number, $\mathrm{U}_{\mathrm{n}}=$ unsigned integer value of the pixel, $\boldsymbol{S}_{\boldsymbol{l}}=$ slope,

$\boldsymbol{I}_{\text {intercept }}=$ intercept.

\section{Cascaded Deep Dilated Residual Network for Liver Segmentation}

\section{Network Architecture}

The proposed DDRN consists of 13 levels in the network. Each level has its distinguishing features (Figure 4). The level L0 is like the first two convolutional layers of VGG net. It is implemented to get the initial feature maps from the image. Level L1 to level L4 is analogous to Res-Net in which skip connection is provided to obtain additional information from previous layers. 


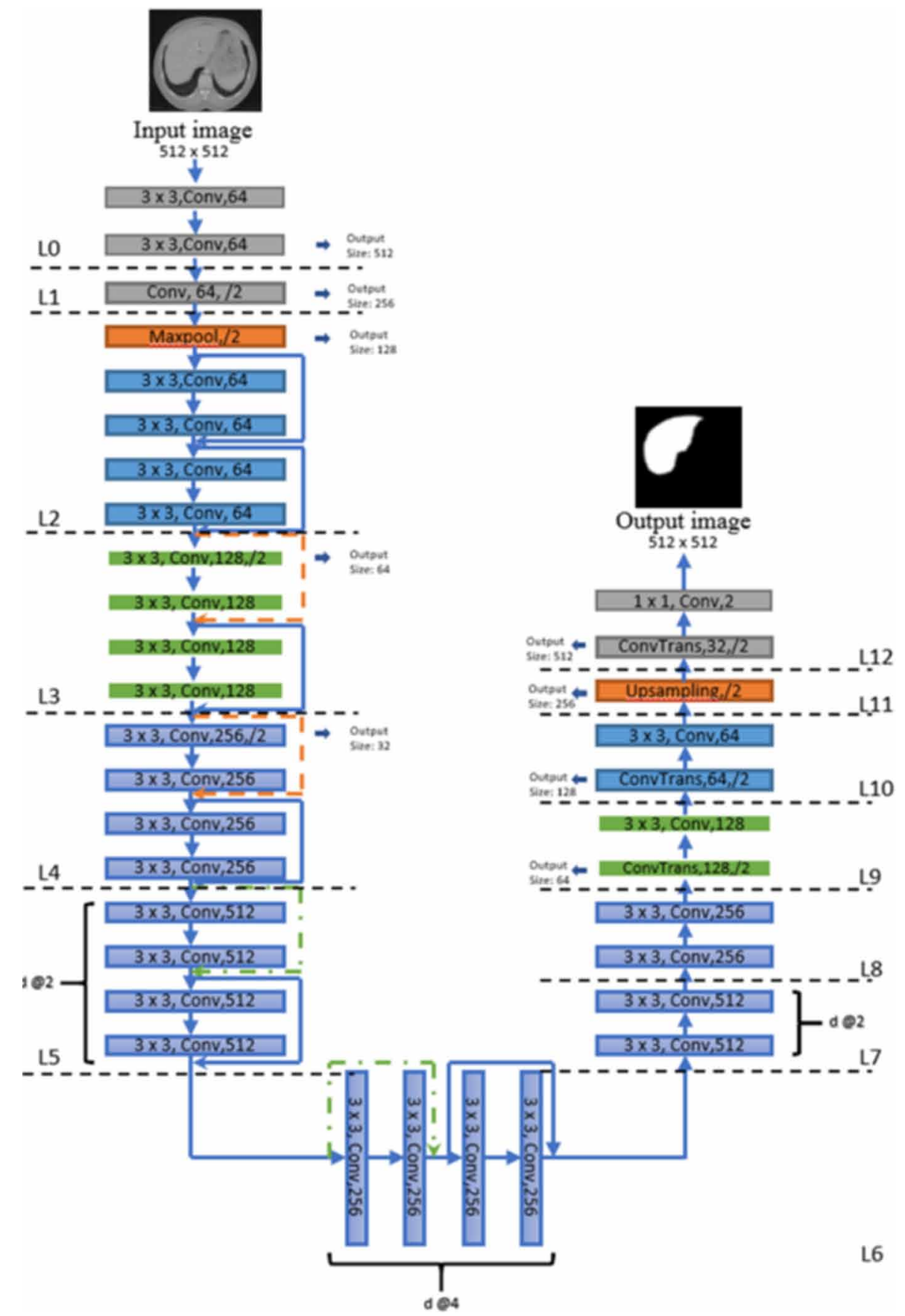

The three types of skip connections are used in this network. Each skip connection comprises of two convolution blocks and one skip connection, as shown in Figure 3, where $x$ is input, $\mathrm{z}$ is output and $\mathrm{y}$ is the intermediate output of the second convolution layer. Input $\mathrm{x}$ is convolved twice at $3 \mathrm{X} 3$ convolution to obtain $\mathrm{y}$. 
Figure 3 (a) is a representation for direct shortcut connection where $x$ is forwarded and added to $\mathrm{y}$ to find $z$. Figure 3 (b) is a representation of a projection shortcut connection, where input $\mathrm{x}$ transformed into $x^{\prime}$ using $1 \times 1$ convolution and stride of 2 . This transformation is required for matching the dimension with intermediate output y for. Projection shortcuts are applied where the output size is decreased. Figure 3 (c) is a representation for the third type of skip connection known as localization shortcut. Localization shortcut $x$ is transformed into $x$ " using $1 \mathrm{X} 1$ convolutions with dilation of two and four(He \& Sun, 2016).

The level L5 to L8 is used dilated residual network for removing gridding artifacts (Yu et al., 2017). Dilation at rate of two is used at level L7, simple convolution at level L8 and eliminated residual shortcuts after level L7. Level L9 to level L12 are decoder of network where number of layers is lesser than U-Net. In decoder, ConvTranspose is used to increase the size of dimensions. The kernel size and its number for various layers of network are shown in Figure 4.

Three Deep dilated Residual Network represented in network architecture are connected in parallel and further these networks are connected with the fourth network in a cascaded manner as shown in Figure 5.

\section{Network Training}

Three DDRN models are trained in order to get the probability map of each model. Original image along with three probability maps of parallel DDRN model is used to train the forth cascaded (Figure 5). CHAOS data sets comprise of 6170 Images of 40 subjects. Network trained using 2570 images and tested on 3296 images. Challenge organizer not revealed ground truth of test data. DDRN weight updated using Stochastic Gradient Descent (SGD), loss function categorical cross-entropy, 40 epochs, batch size set at 5, ReLU activation function and learning rate 0.001 . Network architecture and learnable parameters are kept same for each DDRN. The model is implemented in python using Keras Tensor Flow library in Python platform and trained on NVIDIA GPUs P5000. Single epoch taking 56 minute time while training.

\section{Evaluation Parameters}

Various evaluation parameters are utilized in order to check the performance of medical image segmentation algorithms (Heimann et al., 2009). The evaluation parameters like Dice Coefficient (DICE), Jaccard index (JI), Hausdorff Distance 95 (HD-95), Average Symmetric Surface Distance (ASSD) are calculated for quantitative evaluation of the proposed method (Wu, Zhou, Wu, \& Zhang, 2016). Dice and Jaccard are the segmentation evaluation parameters and ideal range zero to one. Dice value of one shows perfect segmentation with respect to ground truth and zero means segmentation is not perfect. HD-95 and ASSD surface distance between segmented result and ground truth and less values shows perfect volumetric segmentation.

\section{RESULTS AND DISCUSSION}

\section{Preprocessing}

Input CT data windowed in three ranges [-200, 400], [-150, 250] and [-50, 175] abbreviated as W1, W2 and W3 as shown in Figure 6. Windowing operations enhanced contrast compare visuals (Figure 6 (a) to (d)). CT input data divided into three windowed images followed by min-max normalization in order to feed into Deep Dilated Residual Network.

\section{Segmentation Result}

Dilation convolution allows the network to learn at different spatial resolution and windowing helps the network to learn at different gray level quantization, finally enhancing network learning. We have used three patient test data abbreviated as P16, P18, and P28 for depicting the performance of DDRN. 
Figure 5. Schematic of network connection

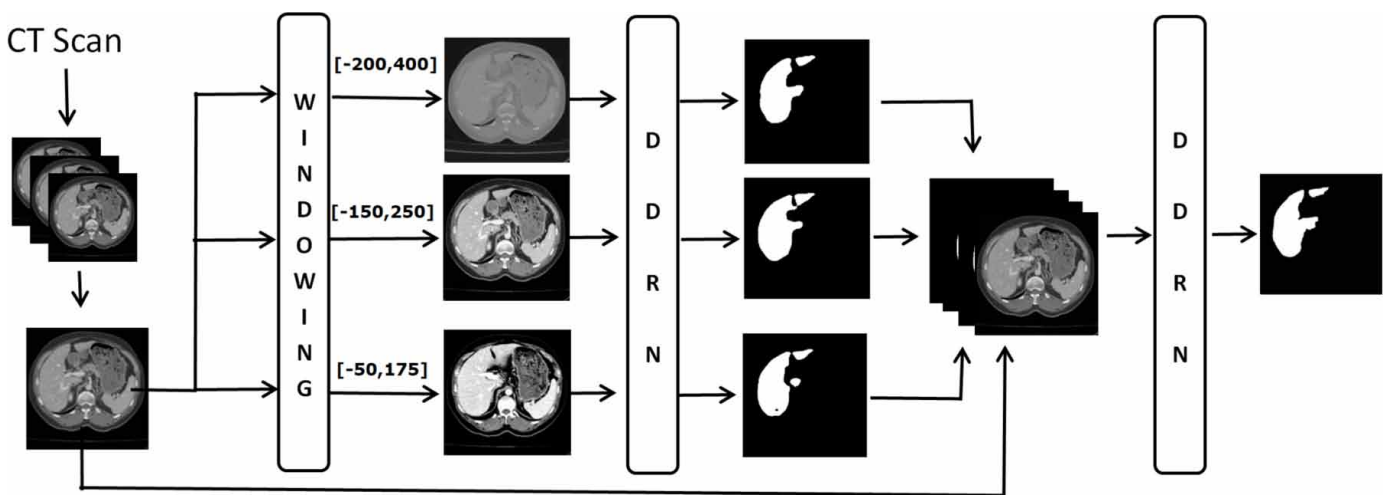

Figure 6. a-d Example of various windowing operation on subject 01 slice sequence 51

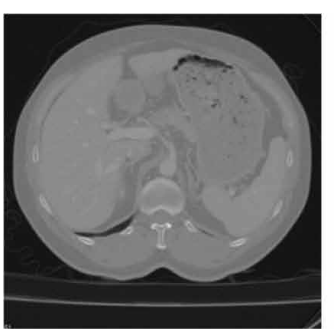

(a) Original Image

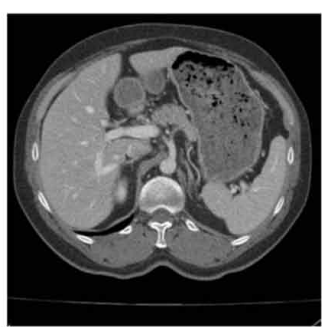

(b) W1 [-200, 400]

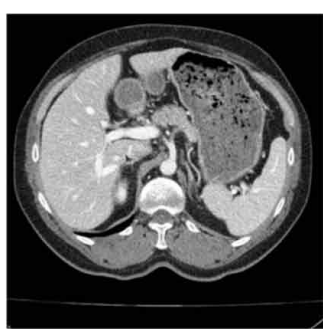

(c) W2 [-150 250]

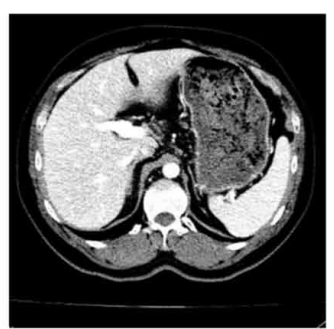

(d) W3 $[-50,170]$

The mean and standard deviation of evaluation parameters are tabulated in Table 1. DICE coefficient does not enhance in cascaded DDRN and shows 0.1 differences compare to average of three windows $\mathrm{W} 1, \mathrm{~W} 2$ and W3 as shown in Table 1. Jaccard index enhances as compared to DICE coefficient for cascaded DDRN. ASSD and HD 95 provide surface differences among the two surfaces. The cascaded network shows less standard deviation and average value for all evaluation parameters compared to all windows. Window W3 performance is superior to W1 and W2 (Table 1). Descriptive statistics of evaluation parameters for W3 and cascaded DDRN are compared for the three test patients (Table 2). Cascaded network performance is far better than $\mathrm{W} 3$ corresponds to each evaluation parameters. Individual window result compared with the cascaded network. It is found that the cascaded network enhanced overall performance in terms of various evaluation matrixes.

Table 1. Comparison of evaluation parameters with respect to W1, W2, W3 and cascaded network

\begin{tabular}{|l|l|l|l|l|}
\hline \multicolumn{1}{|c|}{$\begin{array}{c}\text { Evaluation } \\
\text { Parameter }\end{array}$} & \multicolumn{1}{c|}{$\begin{array}{c}\text { W1 } \\
{[-\mathbf{2 0 0 , 4 0 0 ]}}\end{array}$} & \multicolumn{1}{c|}{$\begin{array}{c}\text { W2 } \\
{[-\mathbf{- 1 5 0 , 2 5 0 ]}}\end{array}$} & $\begin{array}{c}\text { W3 } \\
{[-\mathbf{- 5 0 , 1 7 5 ]}}\end{array}$ & \multicolumn{1}{|c|}{ Cascaded Network } \\
\hline Dice & $0.95 \pm 0.06$ & $0.97 \pm 0.11$ & $0.96 \pm 0.06$ & $0.96 \pm 0.02$ \\
\hline Jaccard & $0.89 \pm 0.14$ & $0.77 \pm 0.17$ & $0.90 \pm 0.13$ & $0.91 \pm 0.14$ \\
\hline HD95 & $32.06 \pm 48.44$ & $90.53 \pm 13$ & $21.59 . \pm 37.07$ & $4.41 \pm 3.45$ \\
\hline ASSD & $4.89 \pm 5.56$ & $15.63 \pm 11.54$ & $3.64 \pm 3.52$ & $1.62 \pm 0.69$ \\
\hline
\end{tabular}


Table 2. Patient bias descriptive statistics for Evaluation Parameter for window W3 verses cascaded network

\begin{tabular}{|c|c|c|c|c|c|c|c|c|c|}
\hline \multirow{2}{*}{$\begin{array}{c}\text { Test } \\
\text { Dataset }\end{array}$} & \multirow{2}{*}{$\begin{array}{c}\text { Descriptive } \\
\text { Statistics }\end{array}$} & \multicolumn{2}{|c|}{ DICE } & \multicolumn{2}{|c|}{ JACCARD } & \multicolumn{2}{|c|}{ ASSD } & \multicolumn{2}{|c|}{ HD95 } \\
\hline & & W3 & $\begin{array}{l}\text { Cascaded } \\
\text { Network }\end{array}$ & W3 & $\begin{array}{l}\text { Cascaded } \\
\text { Network }\end{array}$ & W3 & $\begin{array}{l}\text { Cascaded } \\
\text { Network }\end{array}$ & W3 & $\begin{array}{c}\text { Cascaded } \\
\text { Network }\end{array}$ \\
\hline \multirow[t]{3}{*}{ P16 } & Mean & 0.95 & 0.97 & 0.90 & 0.92 & 4.47 & 4.25 & 24.87 & 1.73 \\
\hline & SD & 0.09 & 0.01 & 0.12 & 0.13 & 4.16 & 1.49 & 37.10 & 0.61 \\
\hline & Median & 0.96 & 0.97 & 0.92 & 0.94 & 2.84 & 4.25 & 6.40 & 1.73 \\
\hline \multirow[t]{3}{*}{ P18 } & Mean & 0.96 & 0.97 & 0.89 & 0.90 & 2.17 & 4.88 & 7.10 & 1.64 \\
\hline & SD & 0.04 & 0.03 & 0.20 & 0.20 & 1.46 & 5.24 & 9.27 & 0.90 \\
\hline & Median & 0.97 & 0.97 & 0.94 & 0.95 & 1.94 & 4.00 & 5.00 & 1.44 \\
\hline \multirow[t]{3}{*}{ P28 } & Mean & 0.97 & 0.97 & 0.93 & 0.94 & 32.82 & 4.05 & 4.31 & 1.51 \\
\hline & SD & 0.01 & 0.01 & 0.02 & 0.01 & 48.33 & 1.72 & 3.82 & 0.36 \\
\hline & Median & 0.97 & 0.97 & 0.94 & 0.94 & 6.00 & 4.00 & 2.06 & 1.48 \\
\hline
\end{tabular}

Box plots of evaluation parameters corresponding to window W1, W2, W3 and cascaded network are shown in Figure 7 (Set-A to Set-D). Each plot is showing three box plots within same figure, which compare results of three subjects. Each patient has an average hundred images. Box plot shows the data distribution; whiskers of the box plot represent a distance from the data median and outliers away from the box area. CT volume data contains average hundreds of slices for individual patients. Dice median corresponds to each patient skewed toward one for the cascaded network; it shows that the cascaded DDRN performed well. In terms of outliers, the cascaded network shows fewer number outliers compared to other window techniques, marked by '+' sign (Figure7, Set-A, (iv)). Falsepositive results always come in the category of an outlier of the box plot. Set-A showing box plot for DICE coefficient with respect to W1, W2, W3, and Cascaded Network, respectively. Number of outlier for W1, W2, W3 and Cascaded Network are 11, 10, 7 and 7, respectively. W3 performance is better among all windows and less compare to cascaded network. Outlier count for W3 and Cascaded Network is same, but the W3 outlier spread range is huge compared to the Cascaded network. All evaluation parameters are consistent for the cascaded network with less number of outliers

We have done critical visual analysis of proposed method while considering images where network was not able to segment accurately. Figure 8 shows the visual result of test subjects along with 3D representations. The result for P16, the network fails to predict concave boundary marked by circle (Figure 8, P16, (c)). 3D model of predicted result helps to highlight the presence false-positive which is difficult in 2D slices. It can be observed in result for P18 mark by square box on 3D predicted result (Figure 8, P18, (d)). The result for P28 shows that 2D and 3D visuals are identical to each other while observing shapes (Figure 8, P28, (b) \& (c)).

We have submitted results of DDRN to the online evaluation system of the CHAOS and system provided evaluation descriptive statistics for Average Symmetric Surface Distance (ASSD), Dice, Maximum Symmetric Surface Distance (MSSD), and Relative Absolute Volume Difference (RAVD) as shown in Table 3. The proposed method got \#\# position on leader board of CHAOS Challenge dated on $22^{\text {nd }}$ October 2019.

\section{CONCLUSION AND FUTURE SCOPE}

In this paper, we proposed a Cascaded DDRN for the automatic segmentation of the liver from CT image data sets. The main contribution of this paper is to evaluate the performance of cascaded DDRN. The extensive experimental results show that the proposed work is good in reducing false-positive results received from different windowing techniques. These methods can save the precious time of 
Figure 7. Box plot for evaluation parameters where (i) W1 [-200,400], (ii) W2, [-150,250], (iii) W3, [-50,175],(iv)Cascaded Network and Set A for DICE, Set B for ASSD, Set C for JI and Set D for HD95

Set A

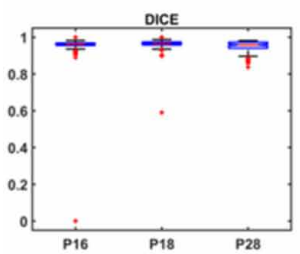

(a) W1 $[-200,400]$

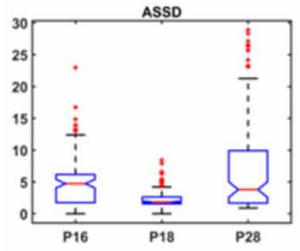

(a) W1 $[-200,400]$

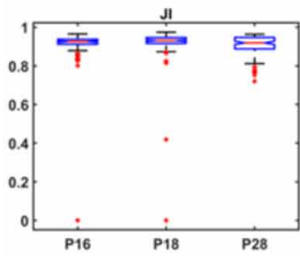

(a) W1 $[-200,400]$

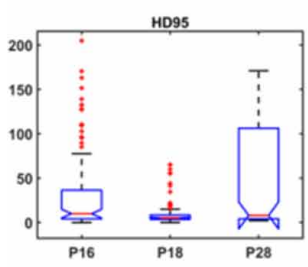

(a) W1 $[-200,400]$

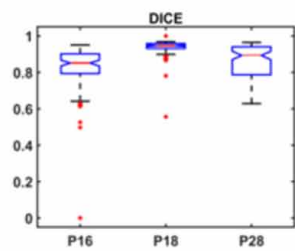

(b) $\mathrm{W} 2,[-150,250]$

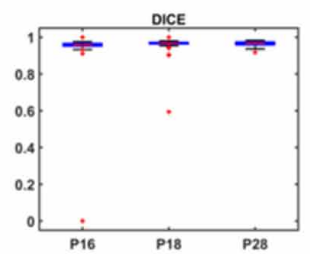

(c) W3, $[-50,175]$

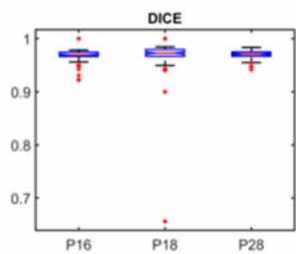

(d)Cascaded Network

Set B

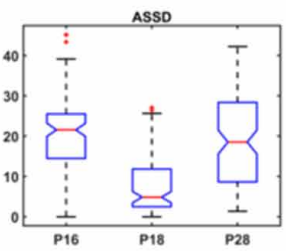

(b) W2 , [-150,250]

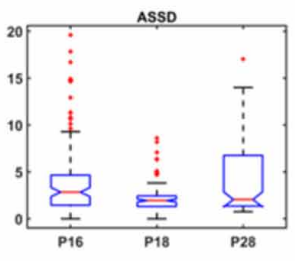

(c) W3, $[-50,175]$

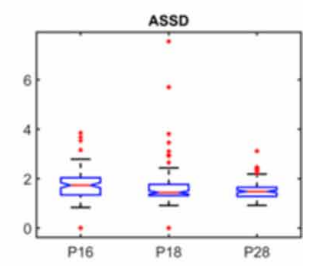

(d)Cascaded Network

Set C

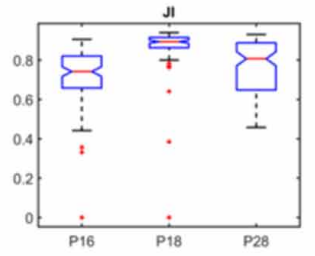

(b) W2 , [-150,250]

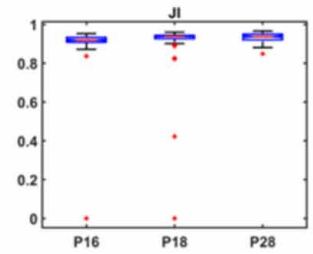

(c) W3, $[-50,175]$

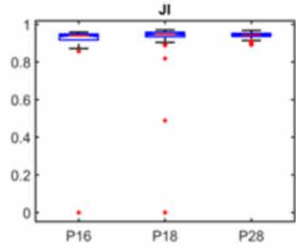

(d)Cascaded Network

Set D

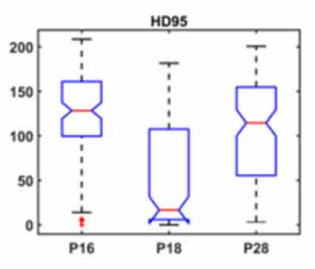

(b) W2 , [-150,250]

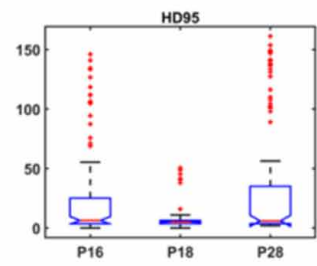

(c) W3, $[-50,175]$

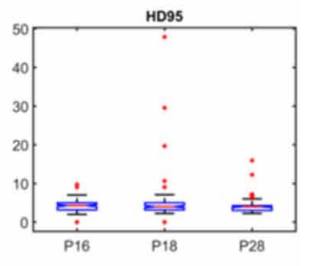

(d)Cascaded Network

medical experts and reduce the waiting queue length. Cascaded Network performance is compared with W1, W2, W3 window DDRN. Windowing selection will remain challenging because it depends upon the area to be targeted, more subjective and depends on window width selection. However, the proposed method has overcome the problem of window dependency and enhances precision. While evaluating the result of the proposed cascaded network we found that a maximum number of slices are showing acceptable results and only a few slices are deviating with respect to their ground truth. This error can be removed while applying 3D connected component post-processing operation. DDRN method yet to be tested on other online data sets because of unavailability CT number gray levels. As future work, these methods will be demonstrated with the clinical application, such as CT guided liver biopsy and radiation therapy planning. 
Figure 8. Visual results for proposed network results

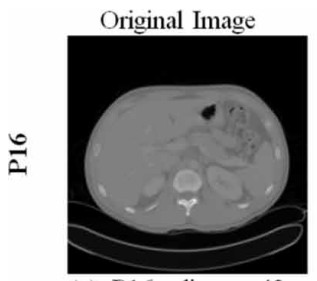

(a). P16_slice no 43

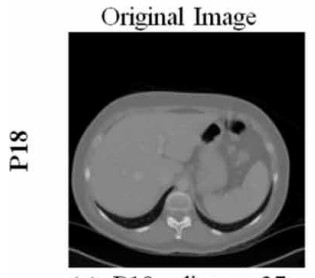

(a). P18_slice no 37

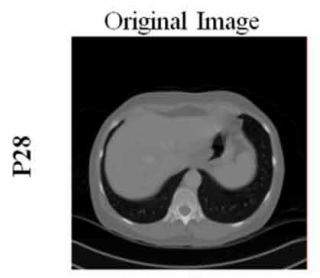

(a). P18 slice no 13

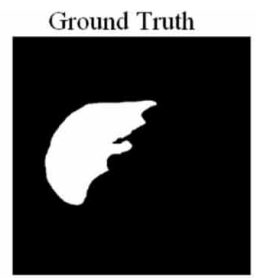

(b). P16_slice no 43

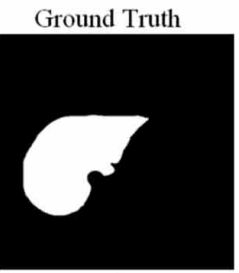

(b). P18_slice no 37

Ground Truth

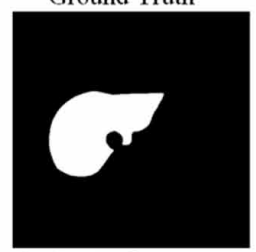

(b). P18 slice no 13

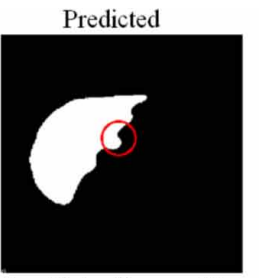

(c). P16_slice no 43

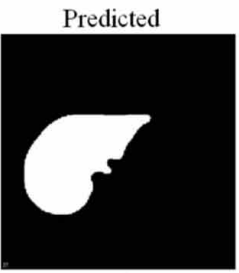

(c). P18_slice no 37

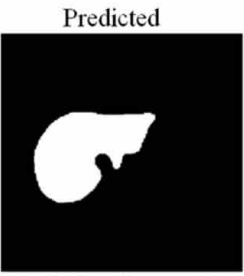

(c). P18 slice no 13

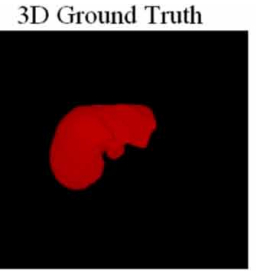

(d). 3D representation

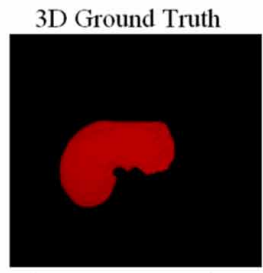

(d). 3D representation

3D Ground Truth

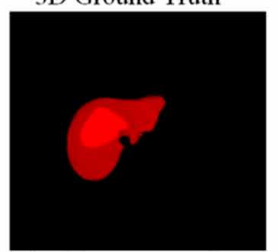

(d). 3D representation
3D Predicted

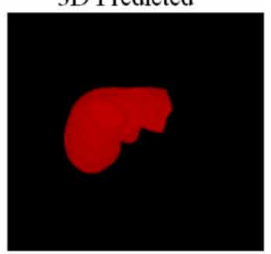

(e). 3D representation

3D Predicted

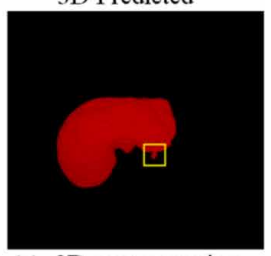

(e). 3D representation

3D Predicted

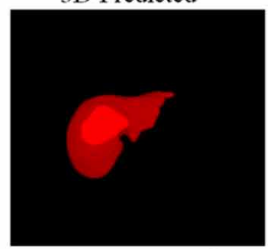

(e). $3 \mathrm{D}$ representation 


\section{REFERENCES}

Asrani, S. K., Devarbhavi, H., Eaton, J., \& Kamath, P. S. (2019). Burden of liver diseases in the world. Journal of Hepatology, 70(1), 151-171. doi:10.1016/j.jhep.2018.09.014 PMID:30266282

Ben-Cohen, A., Diamant, I., Klang, E., Amitai, M., \& Greenspan, H. (2016). Fully convolutional network for liver segmentation and lesions detection. In Deep learning and data labeling for medical applications (pp. 77-85). Springer. doi:10.1007/978-3-319-46976-8_9

Beutel, J., Kundel, H. L., \& Van Metter, R. L. (2000). Handbook of medical imaging (Vol. 1). Spie Press.

Bilic, P., Christ, P. F., Vorontsov, E., Chlebus, G., Chen, H., Dou, Q., ... Menze, B. H. (2019). The Liver Tumor Segmentation Benchmark (LiTS). Retrieved from https://arxiv.org/abs/1901.04056

CHAOS - Combined (CT-MR) Healthy Abdominal Organ Segmentation. (2019). Retrieved December 6, 2019, from https://chaos.grand-challenge.org/Combined_Healthy_Abdominal_Organ_Segmentation/

Conze, P., Groza, V., Pham, D. D., Chatterjee, S., Ernst, P., Baydar, B., ... Maier-hein, K. H. (2020). CHAOS Challenge - Combined (CT-MR) Healthy Abdominal Organ Segmentation. ArXive:2001.06535v1

Glenisson, M., Salloum, C., Lim, C., Lacaze, L., Malek, A., Henriquez, A., \& Azoulay, D. et al. (2014). Accessory liver lobes: Anatomical description and clinical implications. Journal de Chirurgie Viscerale, 151(6), 451-455. doi:10.1016/j.jviscsurg.2014.09.013 PMID:25448768

He, K., \& Sun, J. (2016). Deep Residual Learning for Image Recognition. 2016 IEEE Conference on Computer Vision and Pattern Recognition (CVPR), 770-778. doi:10.1109/CVPR.2016.90

He, K., Zhang, X., Ren, S., \& Sun, J. (2016). Deep residual learning for image recognition. Proceedings of the IEEE Computer Society Conference on Computer Vision and Pattern Recognition, 2016-Decem, 770-778. doi:10.1109/CVPR.2016.90

Heimann, T., Van Ginneken, B., Styner, M. A., Arzhaeva, Y., Aurich, V., Bauer, C., Beck, A., Becker, C., Beichel, R., Bekes, G., Bello, F., Binnig, G., Bischof, H., Bornik, A., Cashman, P., Ying Chi, , Cordova, A., Dawant, B. M., Fidrich, M., \& Wolf, I. et al. (2009). Comparison and evaluation of methods for liver segmentation from CT datasets. IEEE Transactions on Medical Imaging, 28(8), 1251-1265. doi:10.1109/TMI.2009.2013851 PMID:19211338

Lateef, F., \& Ruichek, Y. (2019). Survey on semantic segmentation using deep learning techniques. Neurocomputing, 338, 321-348. doi:10.1016/j.neucom.2019.02.003

Li, W., Jia, F., \& Hu, Q. (2015). Automatic Segmentation of Liver Tumor in CT Images with Deep Convolutional Neural Networks. Journal of Computer and Communications, 03(11), 146-151. doi:10.4236/jcc.2015.311023

Mourya, , Paul, , Handique, , \& Baid, \& Dutande, . (n.d.). Modified U-Net for fully automatic liver segmentation from abdominal CT-image. International Journal of Biomedical Engineering and Technology.

Mourya, G. K., Gogoi, M., \& Handique, A. (2020). Edge Detection Algorithm Based on BEMD for Liver CT Images. International Journal of Innovative Technology and Exploring Engineering, 9(3), 2090-2094. doi:10.35940/ijitee.C8930.019320

Ronneberger, O., Fischer, P., \& Brox, T. (2015). U-net: Convolutional networks for biomedical image segmentation. In International Conference on Medical image computing and computer-assisted intervention (Vol. 9351, pp. 234-241). doi:10.1007/978-3-319-24574-4_28

Shelhamer, E., Long, J., \& Darrell, T. (2017). Fully Convolutional Networks for Semantic Segmentation. IEEE Transactions on Pattern Analysis and Machine Intelligence, 39(4), 640-651. doi:10.1109/TPAMI.2016.2572683 PMID:27244717

Simonyan, K., \& Zisserman, A. (2014). Very Deep Convolutional Networks for Large-Scale Image Recognition. Retrieved from https://arxiv.org/abs/1409.1556

Thapa, B. R., \& Walia, A. (2007). Liver function tests and their interpretation. Indian Journal of Pediatrics, 74(7), 663-671. doi:10.1007/s12098-007-0118-7 PMID:17699976 
WHO. (2012). Indicator Code Book Global Information System on Alcohol and Health. Retrieved October 23, 2019, from https://www.who.int/substance_abuse/activities/gisah_indicatorbook.pdf?ua=1

Wu, W., Zhou, Z., Wu, S., \& Zhang, Y. (2016). Automatic Liver Segmentation on Volumetric CT Images Using Supervoxel-Based Graph Cuts. Computational and Mathematical Methods in Medicine, 2016, 1-14. doi:10.1155/2016/9093721 PMID:27127536

Xue, Z., Antani, S., Long, L. R., Demner-Fushman, D., \& Thoma, G. R. (2012). Window classification of brain CT images in biomedical articles. AMIA ... Annual Symposium Proceedings. AMIA Symposium, 2012, 1023-1029. Retrieved from http://www.ncbi.nlm.nih.gov/pubmed/23304378\%0Ahttp://www.pubmedcentral. nih.gov/articlerender.fcgi?artid=PMC3540547

Yu, F., Koltun, V., \& Funkhouser, T. (2017). Dilated Residual Networks. In 2017 IEEE Conference on Computer Vision and Pattern Recognition (CVPR) (pp. 636-644). IEEE. doi:10.1109/CVPR.2017.75

Gajendra Kumar Mourya (Assistant Professor) Department of Biomedical Engineering, North Eastern Hill University, Shillong. He received his M. Tech in Biomedical Engineering from Indian Institute of Technology (BHU), Varanasi, India. His research interest is Medical Image Processing.

Manashjit Gogoi (PhD) is currently working as an Assistant Professor in the Department of Biomedical Engineering at North-Eastern Hill University, Shillong, India, since 2012. He did B. E. in Chemical Engineering from Assam Engineering College, Guwahati, India and M. Tech. in Bioelectronics from Tezpur University, Tezpur, India. Subsequently, he completed his PhD form Indian Institute of Technology, Bombay, India. Dr. Gogoi is currently working in the fields of nanomedicine and tissue engineering; especially cancer drug delivery, Biosensor and imaging applications. Based on his research work, he published a good number of research papers and book chapters. He is one of the recipients of ABLE-BEST award from Department of Biotechnology (Govt. of India) and Association of Biotechnology Led Enterprises in 2010. He is also reviewer of many internationally reputed journals. Dr. Gogoi has been serving as a Head (i/c) in the Department of Biomedical Engineering, since November, 2018 and Coordinator for the DIC course in the Department of Biomedical Engineering.

Sanjay Talbar (PhD) is a Professor in the Department of Electronics and Telecommunication Engineering, Shri Guru Gobind Singhii Institute of Engineering and Technology, Nanded, Maharashtra, India. He has worked as a Professor and Head at Dr. Babasaheb Ambedkar Technological University, Lonere Raigad, Maharashtra, India from June 2002-May 2004. He has received B.E(Electronics Engineering) and M.E(Electronics Engineering) degrees from SGGS Institute of Technology, Nanded, India in 1985 and 1990 respectively. He obtained his PhD (Electronics and Computer) from SRTM University, Nanded, India in 2000. He received the "Young Scientist Award" by URSI, Italy in 2003. He has supervised about 75 MTech students and $21 \mathrm{PhD}$ students. He is author of seven books, 70 Journal papers and 130 Conference papers. His research interests include Image processing, Biomedical Image Analysis, Computer Vision, Multimedia Computing, Biometrics, and Embedded System Design.

Prasad Dutande received his B.E.(Electronics and Telecommunication Engineering) from Sant Gadge Baba Amravati University, Amravati,Maharashtra, India in 2015, the M. Tech (Electronics and Telecommunication Engineering) from Dr. Babasaheb Ambedkar Technological University, Lonere-Raigad, Maharashtra, India. in 2018. He is currently a Junior Research Fellow at Centre of Excellence in the Department of Electronics and Telecommunication Engineering, Shri Guru Gobind Singhji Institute of Engineering and Technology, Nanded, Maharashtra, India. His research interests include signal processing and image processing.

Ujjwal Baid is a Ph.D. Research Scholar at Center of Excellence in Signal and Image Processing, SGGS Institute of Engineering and Technology, Nanded, Maharashtra, India. He received the B.E. degreein Electronics and Telecommunication Engineering from Amravati University, India, in 2012 and the M. Tech degree from SGGS Institute of Engineering and Technology, Nanded, Maharashtra, India in 2015. He is recipientof prestigious Visvesvaraya fellowship of Government of India towards Ph.D. His major fields of interests are Medical Image Processing, Computer Vision, Machine Learning, Convolutional Neural Networks. 